\title{
Outage Rates and Outage Durations of Opportunistic Relaying Systems
}

\author{
Zoran Hadzi-Velkov, and Nikola Zlatanov
}

\begin{abstract}
Opportunistic relaying is a simple yet efficient cooperation scheme that achieves full diversity and preserves the spectral efficiency among the spatially distributed stations. However, the stations' mobility causes temporal correlation of the system's capacity outage events, which gives rise to its important second-order outage statistical parameters, such as the average outage rate (AOR) and the average outage duration (AOD). This letter presents exact analytical expressions for the AOR and the AOD of an opportunistic relaying system, which employs a mobile source and a mobile destination (without a direct path), and an arbitrary number of (fixed-gain amplify-and-forward or decodeand-forward) mobile relays in Rayleigh fading environment.
\end{abstract}

Index Terms-Average outage rate, Average outage duration, Opportunistic relaying, Doppler effect, Rayleigh fading

\section{INTRODUCTION}

Cooperative diversity is a highly promising technique for coverage extension and reliability improvement of wireless networks. It exploits the additional degrees of freedom of the fading environment, which is introduced by the spatially distributed multiple relays utilizing either amplify-and-forward (AF) or decode-and-forward (DF) relaying. Proposed recently in [1], the opportunistic relaying is a simple yet efficient cooperative diversity protocol, whose diversity-multiplexing tradeoff is identical to that of the more complex distributed spacetime coding cooperative schemes [2]. By selecting a single "best" relay among the all available relays, the opportunistic relaying achieves full spatial diversity while maintaining the spectral efficiency of a two-hop communication link.

The outage and error probabilities of the opportunistic relaying systems have been studied in [3]-[5], which clearly demonstrate its excellent performances. However, there are some design issues for which the outage and error probabilities' criteria are not sufficient, such as, packet or slot lengths and latencies, switching rates [6], power and bandwidth allocation or, decision criterion for changing adaptive modulation levels.

These issues can be addressed by investigating the system's second-order outage statistics. To the best of authors' knowledge, such statistics that describe the outage events of cooperative systems, such as, the average outage rate (AOR) and average outage duration (AOD), have not been studied previously. We propose the AOR and the AOD be defined with respect to the capacity outage events derived from informationtheoretic capacity of the opportunistic system. Similar definition of the outage statistics has been applied over MIMO systems in [7]. In this letter, we derive exact expressions for the AOR and the AOD of opportunistic systems, employing either AF or DF relaying in Rayleigh fading environment.

Accepted for IEEE CommunLetters

Z. Hadzi-Velkov and N. Zlatanov are with the Faculty of Electrical Engineering and Information Technologies, Ss. Cyril and Methodius University, Karpos 2 bb, 1000 Skopje, R. Macedonia, Email: zoranhv@feit.ukim.edu.mk, nzlatanov@manu.edu.mk

\section{OPPORTUNISTIC RELAYING WITH MOBILE STATIONS}

\section{A. System model}

Similarly to [1], we consider a typical half-duplex dual-hop communication scenario, where the communication between the source $S$ and the destination $D$ is possible only via $M$ relays (denoted by $R_{k}, 1 \leq k \leq M$ ), as the direct path is assumed blocked by an intermediate wall. In the beginning of each slot (divided into two equal sub-slots), a single "best" opportunistic relay is selected out of the $M$ possible dualhop paths for relaying the communication between $S$ and $D$. During the first sub-slot, $S$ transmits its signal over the first hop, while the selected relay forwards that signal toward $D$ over the second hop during the second sub-slot.

The channel is exposed to Rayleigh fading and is assumed to remain constant during the entire slot duration. Without loss in generality, we assume that $S$ and the selected "best" relay transmit with equal powers $P_{T}$, rendering the total available transmission power to $2 P_{T}$. Denoting the Rayleighfaded channel gains of hops $S \rightarrow R_{k}$ and $R_{k} \rightarrow D$ during a given slot $t$ by $\alpha_{S k}(t)$ and $\alpha_{k D}(t)$, the received signal-tonoise ratios (SNRs) at the relay $R_{k}$ and at the destination $D$ are expressed as $\gamma_{S k}=P_{T} \alpha_{S k}^{2}(t) / N_{0}$ and $\gamma_{k D}=P_{T} \alpha_{k D}^{2}(t) / N_{0}$, with $N_{0}$ as the noise power. Specifying the average squared channel gains as $E\left[\alpha_{S k}^{2}\right]=\Omega_{S k}$ and $E\left[\alpha_{k D}^{2}\right]=\Omega_{k D}$, the average received SNRs at $R_{k}$ and $D$ are respectively given by $\bar{\gamma}_{S k}=P_{T} \Omega_{S k} / N_{0}$ and $\bar{\gamma}_{k D}=P_{T} \Omega_{k D} / N_{0}$.

The relay selection is based on the estimation of the end-toend performance over the dual-hop path $k$ using the selection variable $W_{k}(t)$, which is estimated separately by each relay $R_{k}$ in the beginning of each slot from its channel state information (CSI) [1]. To facilitate channel state estimation by the relays, $S$ and $D$ previously exchange short control packets. In the beginning of slot $t$, the "best" relay $b$ is selected in a distributed manner by using the selection policy:

$$
b=\underset{1 \leq k \leq M}{\arg \max }\left\{W_{k}(t)\right\} .
$$

1) Decode-and-forward relaying: In the beginning of slot $t$, each DF relay estimates the selection variable

$$
W_{k}^{D F}(t)=\min \left\{\alpha_{S k}(t), \alpha_{k D}(t)\right\},
$$

which actually evaluates the minimal instantaneous received SNR among the two hops, $\min \left\{\gamma_{S k}(t), \gamma_{k D}(t)\right\}$.

2) Amplify-and-forward relaying: We assume fixed gain AF relays that amplify the received signal from the first hop by $\sqrt{P_{T} /\left(P_{T} \Omega_{S k}+N_{0}\right)}$ and forward it to $D$ over the second hop [11]. In the beginning of slot $t$, each $\mathrm{AF}$ relay estimates the selection variable

$$
W_{k}^{A F}(t)=\frac{\alpha_{S k}(t) \alpha_{k D}(t)}{\sqrt{C_{k}+\alpha_{k D}^{2}(t)}},
$$


where $C_{k}=\Omega_{S k}+N_{0} / P_{T}$. Actually, each AF relay evaluates the dual-hop SNR that is relayed over $R_{k}$ and received at $D$. Note that fixed-gain (e.g., semi-blind) AF relays have considerably simpler but yet comparably close performance to that of the variable gain AF relays [11].

\section{B. Stations' mobility}

We consider 2-dimensional isotropic scattering around source $S$, relays $R_{k}$ and destination $D$, all of which are assumed to be mobile and have no line-of-sight with other stations. Thus, each $S \rightarrow R_{k}\left(R_{k} \rightarrow D\right)$ hop behaves as a mobile-to-mobile Rayleigh channel. Its channel gain, $\alpha_{S k}(t)$ $\left(\alpha_{k D}(t)\right)$, is a time-correlated Rayleigh random process with known statistical properties (e.g., the Doppler spectrum) [9]. If a station at one end of a hop is fixed, the mobile-to-mobile Rayleigh-fading hop is transformed into the "classic" fixedto-mobile Rayleigh-fading hop [8]. The time derivative $\dot{\alpha}_{S k}$ $\left(\dot{\alpha}_{k D}\right)$ is independent from the channel gain $\alpha_{S k}\left(\alpha_{k D}\right)$, and follows the Gaussian probability distribution function (PDF) with zero mean and variance [9. Eq. (A5)] [12, Eq. (39)]

$$
\begin{aligned}
\sigma_{\dot{\alpha}_{S k}}^{2} & =\pi^{2} \Omega_{S k}\left(f_{m S}^{2}+f_{m k}^{2}\right), \\
\sigma_{\dot{\alpha}_{k D}}^{2} & =\pi^{2} \Omega_{k D}\left(f_{m k}^{2}+f_{m D}^{2}\right) .
\end{aligned}
$$

In (4)-(5), $f_{m S}, f_{m D}$ and $f_{m k}$ denote the maximum Doppler rates of $S, D$ and relay $R_{k}(1 \leq k \leq M)$, respectively. The Doppler rate (and consequently the AOR) is expressed in the unit slot $^{-1}$. Expressing the slot duration in seconds, both the Doppler rate (now becoming the Doppler frequency) and the AOR are expressed in $\mathrm{Hz}$.

\section{Capacity outage events}

In a given slot $t$, the opportunistic relaying system experiences a capacity outage event when the mutual information of the dual-hop path over the "best" relay $b$ drops below some predefined spectral efficiency $R$ [2],

$$
I(t)=\frac{1}{2} \log _{2}\left(1+\frac{P_{T}}{N_{0}}\left(W_{\max }(t)\right)^{2}\right) \leq R,
$$

where

$$
W_{\max }(t)=\max \left\{W_{1}(t), W_{2}(t), \ldots, W_{M}(t)\right\} .
$$

Thus, the resulting time-varying capacity $I(t)$ suffers from the random occurrence of capacity outage events, during which the channel is unable to support the specified $R$. Transforming (6), the capacity outage event at slot $t$ occurs if

$$
W_{\max }(t) \leq Z \text {, }
$$

where the outage threshold is $Z=\sqrt{\left(2^{2 R}-1\right) /\left(P_{T} / N_{0}\right)}$. Note, $Z$ can be varied by varying $R$ (as in [7]) or $P_{T} / N_{0}$ (as in this work), but the functional dependencies of the increasing $R$ or the decreasing $P_{T} / N_{0}$ (in $\mathrm{dB}$ ) have almost same shapes.

\section{Average Outage Rates and Outage Durations}

\section{A. General expression}

Using a similar approach to that presented in [10] for deriving level crossing rates (LCR) of "classic" selection diversity systems, the joint PDF of $W_{\max }$ and $\dot{W}_{\max }$ is expressed as

$$
f_{W_{\max } \dot{W}_{\max }}(w, \dot{w})=\sum_{k=1}^{M} f_{W_{k} \dot{W}_{k}}(w, \dot{w}) P_{k}(w)
$$

where $f_{W_{k} \dot{W}_{k}}(w, \dot{w})$ denotes the joint PDF of selection variable $W_{k}(t)$ and its time derivative $\dot{W}_{k}(t)$. Assuming $R_{k}$ is selected "best" relay, $P_{k}(w)$ denotes the conditional probability that the selection variable $W_{k}$ of $R_{k}$ drops below $w$,

$$
P_{k}(w)=\operatorname{Pr}\left\{W_{k} \leq w \mid W_{k} \text { is } \max \right\}=\prod_{i=1, i \neq k}^{M} F_{W_{i}}(w),
$$

where $F_{W_{i}}(w)=\operatorname{Pr}\left\{W_{i} \leq w\right\}$ denotes the cumulative distribution function (CDF) of $W_{i}$. The AOR is evaluated based on the standard LCR definition [8, Chapter 1], yielding

$$
\begin{aligned}
& N(Z) \stackrel{\text { def }}{=} \int_{0}^{\infty} \dot{w} f_{W_{\max } \dot{W}_{\max }}(Z, \dot{w}) d \dot{w} \\
& =\sum_{k=1}^{M} P_{k}(Z) \int_{0}^{\infty} \dot{w} f_{W_{k}, \dot{W}_{k}}(Z, \dot{w})=\sum_{k=1}^{M} P_{k}(Z) N_{k}(Z),
\end{aligned}
$$

where $N_{k}(Z)$ denotes the AOR of the dual-hop path over the relay $R_{k}$. The AOD is then given by

$$
\begin{aligned}
T(Z) \stackrel{\text { def }}{=} & \frac{\operatorname{Pr}\left\{W_{\max } \leq Z\right\}}{N(Z)} \\
& =N(Z)^{-1} \prod_{k=1}^{M} F_{W_{k}}(Z)=\left(\sum_{k=1}^{M} \frac{N_{k}(Z)}{F_{W_{k}}(Z)}\right)^{-1} .
\end{aligned}
$$

\section{B. Decode-and-forward relaying}

We now focus on the random process $W_{k}^{D F}(t)$, defined by (2). Communication through the relay $R_{k}$ falls in outage if either one of the two hops fail. Thus, the PDF of $W_{k}^{D F}(t)$ is given by

$$
f_{W_{k}}(w)=f_{\alpha_{S k}}(w) \operatorname{Pr}\left\{\alpha_{k D}>w\right\}+f_{\alpha_{k D}}(w) \operatorname{Pr}\left\{\alpha_{S k}>w\right\} .
$$

Since both hops follow the Rayleigh PDF, $W_{k}^{D F}$ is also determined to follow the Rayleigh PDF,

$$
f_{W_{k}}(w)=2 \Lambda_{k} w e^{-\Lambda_{k} w^{2}}
$$

with $\Lambda_{k}=\Omega_{S k}^{-1}+\Omega_{k D}^{-1}$, and the respective $\operatorname{CDF} F_{W_{k}}(w)=$ $1-\exp \left(-\Lambda_{k} w^{2}\right)$. Using

$$
\dot{W}_{k}=\left\{\begin{array}{cc}
\dot{\alpha}_{S k}, & \alpha_{S k} \leq \alpha_{k D} \\
\dot{\alpha}_{k D}, & \alpha_{k D}<\alpha_{S k}
\end{array}\right.
$$

and the independence of the channel gains and their respective time derivatives, the required joint PDF is found as

$$
\begin{aligned}
f_{W_{k} \dot{W}_{k}}(w, \dot{w}) & =f_{\dot{\alpha}_{S k}}(\dot{w}) f_{\alpha_{S k}}(w) \operatorname{Pr}\left\{\alpha_{k D}>w\right\} \\
& +f_{\dot{\alpha}_{k D}}(\dot{w}) f_{\alpha_{k D}}(w) \operatorname{Pr}\left\{\alpha_{S k}>w\right\} .
\end{aligned}
$$

In Rayleigh fading, (16) specializes to

$$
f_{W_{k} \dot{W}_{k}}(w, \dot{w})=f_{W_{k}}(w) f_{\dot{W}_{k}}(\dot{w}),
$$

thus rendering $W_{k}$ and $\dot{W}_{k}$ as independent RVs, where $f_{W_{k}}(w)$ is given by (14) and

$$
f_{\dot{W}_{k}}(\dot{w})=\frac{\Omega_{k D}}{\Omega_{S k}+\Omega_{k D}} f_{\dot{\alpha}_{S k}}(\dot{w})+\frac{\Omega_{S k}}{\Omega_{S k}+\Omega_{k D}} f_{\dot{\alpha}_{k D}}(\dot{w})
$$




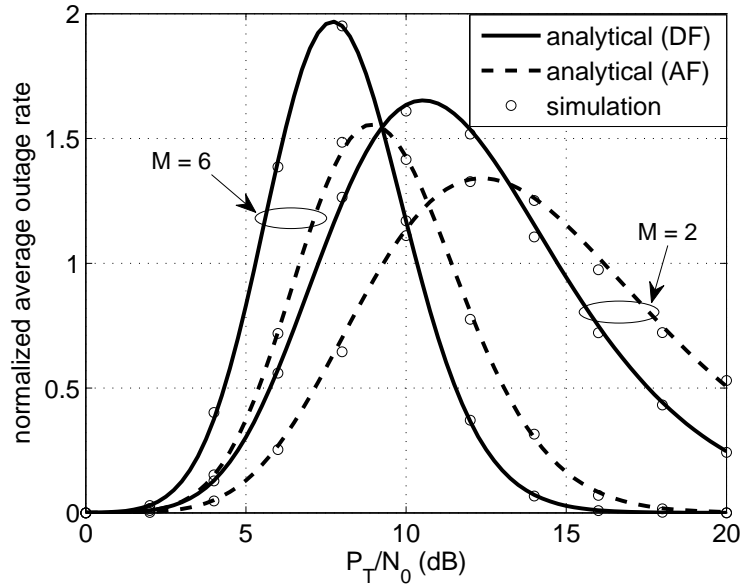

Fig. 1. Normalized Average Outage Rate in DF and AF opportunistic relaying systems

with $f_{\dot{\alpha}_{S k}}(\cdot)$ and $f_{\dot{\alpha}_{k D}}(\cdot)$ denoting zero mean Gaussian PDFs with variances $\sigma_{\dot{\alpha}_{S k}}^{2}$ (4) and $\sigma_{\dot{\alpha}_{k D}}^{2}$ (5), respectively. Thus, the AOR of dual-hop path over $R_{k}$ is obtained as

$$
N_{k}(Z)=\frac{\Omega_{k D} \sigma_{\dot{\alpha}_{S k}}+\Omega_{S k} \sigma_{\dot{\alpha}_{k D}}}{\Omega_{S k}+\Omega_{k D}} \frac{f_{W_{k}}(Z)}{\sqrt{2 \pi}}
$$

with $f_{W_{k}}(\cdot)$ given by (14). Inserting (19) into (11) and (12), we obtain the AOR and the AOD of DF relaying system.

\section{Amplify-and-forward relaying}

The exact expressions for the CDF and the AOR of the random process $W_{k}^{A F}(t)$, defined by (3), are respectively given by [11, Eq. (9)] and [12, Eq. (19)], as

$$
\begin{aligned}
F_{W_{k}}(Z)=1-2 Z \sqrt{\frac{C_{k}}{\Omega_{S k} \Omega_{k D}}} \exp \left(-\frac{Z^{2}}{\Omega_{S k}}\right) \\
\times K_{1}\left(2 Z \sqrt{\frac{C_{k}}{\Omega_{S k} \Omega_{k D}}}\right), \\
N_{k}(Z)=\sqrt{\frac{2}{\pi}} \frac{2 Z \exp \left(-Z^{2} / \Omega_{S k}\right)}{\Omega_{S k} \Omega_{k D}} \\
\times \int_{0}^{\infty} \sqrt{\sigma_{\dot{\alpha}_{S k}}^{2}\left(y^{2}+C_{k}\right)+\frac{1}{y^{4}} \sigma_{\dot{\alpha}_{k D}}^{2} C_{k}^{2} Z^{2}} \\
\quad \times \exp \left(-\frac{\Omega_{S k} y^{4}+C_{k} \Omega_{k D} Z^{2}}{y^{2} \Omega_{S k} \Omega_{k D}}\right) d y,
\end{aligned}
$$

where $K_{1}(\cdot)$ is the first-order modified Bessel function of the second kind. Note that (21) can be efficiently and accurately evaluated by applying the Gauss-Hermite quadrature rule [13, Eq. (25.4.46)]. Combining (20) and (21) into (11) and (12), we obtain the AOR and the AOD of AF relaying system.

Note, if source $S$ and destination $D$ are fixed, the approach presented in this section can be applied to derive analogous analytic expressions for AORs and AODs for more general fading channels (such as, Rice and Nakagami- $m$ models), because such fixed-to-mobile hops have known second-order statistical properties.

\section{NUMERICAL AND SIMULATION EXAMPLES}

In this section, we present illustrative examples for the normalized AOR (Fig. 1) an the normalized AOD (Fig. 2) in

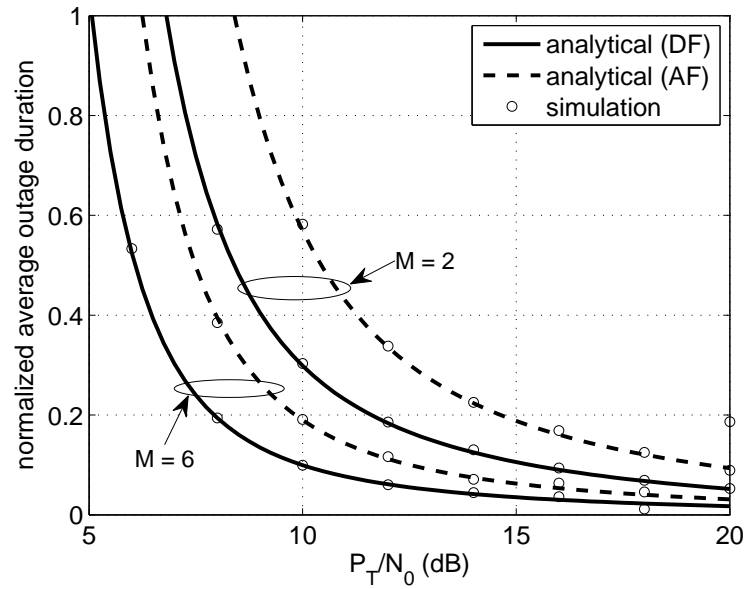

Fig. 2. Normalized Average Outage Duration in DF and AF opportunistic relaying systems

function of $P_{T} / N_{0}$ of opportunistic relaying system employing either DF or AF relays. The source $S$ and the destination $D$ are fixed $\left(f_{m S}=f_{m D}=0\right)$, whereas all the relays are mobile and introduce same maximum Doppler rates $f_{m k}=f_{m 0}$, $1 \leq k \leq M$. The AOR and the AOD are normalized with respect to the Doppler rate $f_{m 0}$ as $N / f_{m 0}$ and $T \cdot f_{m 0}$. The average squared channel gains of all hops are equal to 0.5 (i.e., $\Omega_{S k}=\Omega_{k D}=0.5,1 \leq k \leq M$ ), thus rendering total available transmission power equal to $P_{T}$. The spectral efficiency is set to $R=1 \mathrm{bps} / \mathrm{Hz}$. The Monte Carlo simulations clearly validate our derived analytical results.

\section{REFERENCES}

[1] A. Bletsas, A. Khisti, D. P. Reed and A. Lippman, "A simple cooperative diversity method based on network path selection," IEEE J. Select. Areas. Commun., vol. 24, no. 3, pp. 659-672, Mar. 2006

[2] J. N. Laneman and G. W. Wornell, "Distributed space-time coded protocols for exploiting cooperative diversity in wireless networks," IEEE Trans. Inform. Theory, vol. 49, no. 10, pp. 2415-2525, Oct. 2003

[3] A. Bletsas, H. Shin, and M. Z. Win, "Cooperative communications with outage-optimal opportunistic relaying," IEEE Trans. Wireless Commun., vol. 6, no. 9 , pp. $3450-3460$, Sept. 2007

[4] D. S. Michalopoulos and G. K. Karagiannidis, "Performance analysis of single relay selection in Rayleigh fading", IEEE Trans. Wireless Commun., vol. 7, no. 10, pp. 3718-3724, Oct. 2008

[5] K.-S. Hwang, Y.-C. Ko, and M.-S. Alouini, "Outage probability of cooperative diversity systems with opportunistic relaying based on decodeand-forward", IEEE Trans. Wireless Commun., vol. 7, no. 12, Dec. 2008

[6] D. Michalopoulos, A. Lioumpas, G. K. Karagiannidis and R. Schober, "Selective cooperative relaying over time-varying channels", submitted to IEEE Trans. Commun., http://arxiv.org/abs/0905.0564v1

[7] B. O. Hogstad, M. Patzold, N. Youssef, V. Kontorovitch, "Exact closedform expressions for the distribution, the level-crossing rate, and the average duration of fades on the capacity of OSTBC-MIMO channels", IEEE Trans. Veh. Technol., vol. 58, no. 2, pp. 1011-1016, Feb. 2009

[8] W. C. Jakes, Microwave Mobile Communications, 2nd ed. Piscataway, NJ: IEEE Press, 1994.

[9] A. S. Akki and F. Haber, "A statistical properties of mobile-to-mobile land communication channel," IEEE Trans. Veh. Technol., vol. 43, no. 4, pp. 826-831, Nov. 1994

[10] X. Dong and N. C. Beaulieu, "Average level crossing rate and average fade duration of selection diversity", IEEE Commun. Letters, vol. 5, no. 10, Oct. 2001

[11] M. O. Hasna and M.-S. Alouini, "A performance study of dual-hop transmissions with fixed gain relays", IEEE Trans. Wireless Commun., vol. 3, no. 6, pp. 1963-1968, Nov. 2004

[12] C.S. Patel, G.L. Stuber and T.G. Pratt, "Statistical properties of amplify and forward relay fading channels," IEEE Trans. Veh. Technol., vol. 55, no. 1, pp. 1-9, Jan. 2006 
[13] M. Abramowitz and I. A. Stegun, Handbook of Mathematical Functions with Formulas, Graphs, and Mathematical Tables, 9th ed. New York: Dover, 1970 